\title{
THE ROLE OF RETAILER'S APP CHANNEL IN A SUPPLY CHAIN WITH QUALITY DECISION AND DIFFERENT POWER STRUCTURES
}

\author{
Musen XuE*
}

\begin{abstract}
The role of retailer's ability to add app channel in a supply chain with quality decision and different power structures is investigated in this paper. Applying a game-theoretic approach, we find that, first, under certain conditions, retailer's ability to add app channel can induce the manufacturer to adjust the wholesale price and product quality in the opposite direction with the manufacturer being the leader. Second, for the manufacturer and the retailer, retailer's ability to add app channel can result in two distinct profit situations regardless of the power structure of supply chain: win-win and lose-win. Moreover, in a retailer-led supply chain, adding app channel will make the whole supply chain better off when the return cost is relatively low or high, while make the whole supply chain worse off when the return cost is moderate. Third, we identify a region of the return cost under which the manufacturer, the retailer, the supply chain and consumers can gain from adding app channel, leading to a Pareto improvement.
\end{abstract}

Mathematics Subject Classification. 90B50, 91A80.

Received August 24, 2019. Accepted February 19, 2020.

\section{INTRODUCTION}

\subsection{Motivation and research questions}

In 2017, the online retail momentum of China approaches $\$ 1$ trillion due to the rapid development of Internet technologies and e-commerce. Increasing mobile penetration will also boost online sales [41]. The universal e-commerce and mobile penetration have induced many retailers to open app channel to sell product online in the final market. For example, Leyou, as the largest for baby products and one of China's leading speciality retailers, has developed app to provide product through online channel. The app channel not only provides information about product but also builds a community for mothers. In addition, app shopping allows repeat returns and enhances Leyou's brand perception [6]. However, consumers have to undertake some hassle costs which are caused by the process of product return, such as money penalties for each return product, restocking fees or delivery payments [30].

It is an effective way for the retailer to grab profit through establishing app channel along with physical channel because it can reach many potential customers. The app channel may compete with physical stores and cannibalize the business of offline channel [2]. However, adding app channel enables the large

Keywords. App channel, power structure, supply chain, game theory.

School of Management, Tianjin Normal University, Tianjin 300387, P.R. China.

*Corresponding author: msxue@tju.edu.cn 
bricks-and-mortar retailer to bring benefit. As indicated by Wallace et al. [34], comparing with a single-channel retailer, a dual-channel retailer can enhance customers' satisfaction and ultimately customers' loyalty. In addition, product quality, as an important decision, plays a significant role in product design and inventory strategies of a supply chain [22-24]. Firms' product quality and pricing strategies have been significantly affected by channel structures $[9,15,33,35]$. Different levels of product quality may be induced by different channel structures to be employed. As a result, a question is naturally raised that how the retailer's ability to add app channel affects pricing and product quality decisions.

The power structure of supply chain has been changed by the fast advances in Internet technologies. Traditionally, the supply chain is controlled by the manufacturers. However, this knowledge has been reversed by the power retailers. The performance of supply chain is significant affected by the emergence of power retailers [28]. For the supply chain members who target at maximizing their own profits, power structure is expressed by ability of controlling the process of decision making in a supply chain. To the best of our knowledge, the role of retailer's ability to open an app channel with different power structures of supply chain remains unexplored.

Based on above discussion, the study tries to investigate the effects of adding app channel on the equilibrium outcomes of chain members under different power structures' setting, and intent to address the research questions as follows:

(1) How does retailer's ability to open app channel affect the equilibrium pricing and product quality decisions under different power structures' setting in a supply chain?

(2) What are the impacts of adding app channel on the profits of the channel members and total supply chain, as well as consumer surplus under different power structures' setting?

(3) Under what conditions can adding app channel lead to a Pareto improvement for the channel members, total supply chain and consumers?

\subsection{Main results and contribution}

To address the above-mentioned questions, we consider a supply chain consisting of a manufacturer and a retailer. The manufacturer sets the product quality level and wholesale price. The retailer resells product procured from the manufacturer. The retailer has ability to open app channel to sell product online. If the retailer opens an app channel, it engages in channel conflicts in the retail market. With setting different power structures of supply chain and applying a game-theoretic approach, we obtain the equilibrium outcomes and gain some novel managerial insights as follows:

(1) We find that the impacts of adding app channel on the strategies of supply chain members under different power structures of supply chain are quite different. When the return cost is sightly high, adding app channel can induce the manufacturer to adjust the wholesale price and product quality in the opposite direction in a manufacturer-led supply chain. However, the trend of adjusting the wholesale price and product quality with adding app channel is always consistent in a retailer-led supply chain. (2) For the manufacturer and the retailer, retailer's ability to add app channel can lead to two distinct profit situations regardless of power structure: winwin and lose-win. We demonstrate that adding app channel is able to make the manufacturer better off without any transfer payment. In addition, it is more likely to result in a win-win situation with adding app channel in a retailer-led supply chain. From the whole supply chain's perspective, adding app channel always makes the whole supply chain better off when the manufacturer acts the leader of the supply chain. In a retailer-led supply chain, however, the results are conditional on the return cost. The whole supply chain will gain from adding app channel when the return cost is relatively low or high, while the whole supply chain will be suffered from adding app channel when the return cost is moderate. (3) A new result is presented to identify a region in the return cost where the manufacturer, the retailer, the supply chain and consumers will simultaneously gain from adding app channel, which means that adding app channel is able to result in a Pareto improvement. Specifically, when the return cost is neither too small nor too large, it is possible for all parts (the manufacturer, the retailer, the supply chain and consumers) to reach a Pareto improvement from adding app channel when the manufacturer acts the leader. However, in a retailer-led supply chain, all the parts (the manufacturer, the retailer, the supply 
chain and consumers) can not simultaneously become better off from retailer's ability to add app channel. All in all, manager of the retailer should take the return cost into serious consideration because the introduction of app channel can potentially affect the performance of chain members and consumers.

We summary the contributions of this study as follows. First, this research contributes to the existing literature by investigating the price and quality strategies with retailer opening app channel. Second, we not only derive the equilibrium outcomes for chain members under different power structures of supply chain, but also analyse the impacts of adding app channel on the profits of channel members and total supply chain, as well as consumer surplus. Third, managerial insights obtained in this paper can provide rules for manufacturer and retailer to develop appropriate pricing and quality strategies with retailer's ability to open app channel and the specific power structures of supply chain

The remainder of this paper is organized as follows. In Section 2, we review the existing literature that is related to our study. In Section 3, we give the basic model. Also, the equilibrium outcomes with manufacturer being the leader and retailer being the leader are derived, respectively. In Section 4, we compare the equilibrium outcomes of different subgames. In Section 5, we investigate the effect of adding app channel on consumer warfare. Section 6 provides a conclusion of this paper.

\section{LITERATURE REVIEW}

Literature related to this study comes from the following two streams: retailer's direct channel strategy and power structure in supply chain management.

Recent studies have paid attention to the retailer's channel strategy. Huang and Swaminathan [13] examine the optimal pricing strategy for a retailer who opens an Internet channel along with a traditional channel. The effects of new alternative channels on prices and profits with competition are also discussed. Avery et al. [4] examine how adding a new retail store channel affects the existing direct channel. Niu et al. [25] consider the problem of joint pricing and production decisions in a supply chain with the retailer operating a dual-channel. Their results show that, for a dual-channel retailer, the pricing decision in one channel generates effects on the pricing and production decisions in the other channel. Li and Liu [19] investigate the pricing policy with retailer opening direct channel, in which the decisions are made on the basis of decentralized and centralized supply chains. Tao et al. [32] examine how delivery options affect a local seller's app channel strategy. They propose a joint pricing and delivery distance decision model, and the optimal decisions for the local seller's are derived. Considering retailer acting the leader of the supply chain, Zhang et al. [46] study a retailer's channel strategy and pricing decision. Niu et al. [26] examine how the uniform pricing and online-to-store channel affect traffic congestion control. The main difference between our study and the existing literature is that we investigate the role of app channel in a supply chain from the retailer's point of view. Moreover, the product quality is considered as an endogenous decision under different power structures setting in a supply chain, which leads to different results.

Another stream related to our study is the literature studying power structure in supply chain. In the presence of two dual-exclusive channels, Zhang et al. [43] examine how products' substitutability and channel position affect the pricing decision in different power structures. The effect of power structure with random demand on the performance of supply chain members is examined in Shi et al. [28]. Xue et al. [39] investigate the impacts of power schemes on the supply chain members' performance and consumer surplus. With different power structures' setting, Chen et al. [8] study a retail service supply chain with retailer operating online-to-offline channel. In contrast to the above papers, this research, from retailers' perspective, focuses on investigating the effects of adding app channel on the equilibrium outcomes of chain members under different power structures' setting. 
TABLE 1. List of notations.

\begin{tabular}{ll}
\hline \hline Symbol & Description \\
\hline Indices & The manufacturer's profit \\
$\pi_{m}$ & The retailer's profit \\
$\pi_{t}$ & The demand without app channel \\
$D$ & The demand for physical channel with app channel \\
$D_{t}$ & The demand for app channel \\
$D_{a}$ & Consumers' reservation value \\
Parameters & A probability that consumers like the product purchased from app channel \\
$v$ & Consumers' return cost \\
$\theta$ & \\
$r$ & The wholesale price \\
Decision variables & The product quality \\
$u$ & The selling price of physical channel \\
$p_{t}$ & The selling price of app channel \\
$p_{a}$ &
\end{tabular}

\section{MODEL FORMULATiON}

\subsection{Notations}

The following notations are employed to formulate our model. We give the notations in Table 1.

\subsection{The model}

In this section, the basic model is formulated. A supply chain consisting of a manufacturer and a retailer is considered. The manufacturer sells product to a retailer by charging a wholesale price $w$, and the retailer sets selling price $p_{t}$ to sell the product to final consumers. Without loss of generality, the production cost is supposed to be zero [48]. The retailer may open its own app channel to sell product online. The manufacturer also determines product quality level represented by $u$ with a cost $c(u)$. The cost $c(u)$ is supposed to take a quadratic function of the quality level $u$, i.e., $c(u)=\frac{1}{2} u^{2}$. Such quadratic function implies the increasing marginal costs of quality improvement, which is popularly used in the literature, e.g., Chen [7], Shi et al. [27], Zhang et al. [44] and Dai et al. [11].

Following Xu et al. [36], Yu et al. [40] and Xue and Zhang [37], it is supposed that each consumer purchases at most one unit of the product as long as his/her utility is positive. The market size is normalized to 1 without loss of generality. Let $v$ represent the base value of the product $[16,18]$. We suppose that the product's value $v$ is uniformly distributed in $[0,1]$. This assumption is common in the literature, such as Zhang and Cooper [42], Liu and Shum [20], Shum et al. [31] and Zhu and Xue [49]. Similar to Kuksov and Lin [18], the utility of a consumer buying through the tailer channel is $U_{t}=v+u-p_{t}$. If the retailer does not establish the app channel, a consumer will buy through the physical channel if $U_{t}>0$ is satisfied. Thus, we can obtain the product demand without app channel as

$$
D=1+u-p_{t}
$$

If the retailer establishes an app channel, he sets a selling price $p_{a}$ to sell product through this online channel. When purchasing from the app channel, consumers aren't able to physically inspect and possess the product immediately. Thus, we use $\theta(0<\theta<1)$ to represent a probability that a consumer likes the product obtained from the app channel. The app channel allows consumers to return the disliked product. The expected utility of consumers from buying the product through the app channel can be expressed as $U_{a}=\theta\left(v+u-p_{a}\right)-(1-\theta) r$. $r$ represents an extra hassle cost for returning the product if consumers don't like the product purchased from 
the app channel. As $\theta$ increases, the products purchased from the app channel give consumer more utility. The utility function $U_{a}$ reflects that consumers obtain a utility $v+u-p_{a}$ from the product if she likes it, and a utility of $-r$ otherwise. The similar expected utility function is commonly applied in the literature, such as Balakrishnan et al. [5], Jing [17] and Mehra et al. [21]. An assumption is proposed to ensure positive equilibrium strategies and profits, that is $0<r<\frac{\theta}{1-\theta}$. The similar assumption is commonly employed in the literature, such as Shulman and Geng [29], Dai et al. [11] and Zhang et al. [47].

From the utility functions discussed above, consumers' choices between the selling channels are on the bases of their utility. A consumer will buy from the physical channel when the following conditions are satisfied

$$
\left\{\begin{array}{l}
v+u-p_{t}>0 \\
v+u-p_{t}>\theta\left(v+u-p_{a}\right)-(1-\theta) r .
\end{array}\right.
$$

Analogously, a consumer will purchase through the app channel when the following conditions are satisfied

$$
\left\{\begin{array}{l}
\theta\left(v+u-p_{a}\right)-(1-\theta) r>0 \\
\theta\left(v+u-p_{a}\right)-(1-\theta) r>v+u-p_{t} .
\end{array}\right.
$$

We can obtain the demand expressions for the physical channel and app channel, respectively, which are given as

$$
\begin{aligned}
& D_{t}=1-\frac{p_{t}-\theta p_{a}-(1-\theta) r+(\theta-1) u}{1-\theta} \\
& D_{a}=\frac{p_{t}-\theta p_{a}-(1-\theta) r+(\theta-1) u}{1-\theta}-\frac{\theta p_{a}+(1-\theta) r-\theta u}{\theta} .
\end{aligned}
$$

We define two different power structures in a supply chain to investigate how establishing app channel interacts with different power structures. According to El-Ansary and Stern [12], supply chain member's power can be expressed by its ability to control the decision variables of another member. We follow this definition in this study. Different decision sequences determined by the manufacturer and the retailer are employed to represent different power structures. Specifically, the manufacturer and retailer Stackelberg games are applied to model the supply chain dominated by the manufacturer and retailer, respectively.

\subsection{Manufacturer-led stackelberg}

In this section, we consider the case that the manufacturer is the leader of supply chain, meaning the manufacturer acts as a Stackelberg leader, and the retailer responds as a Stackelberg follower. Let $f$ denote this case. The sequence of events is as follows: (i) the manufacturer sets the quality level $u$ and wholesale price $w$; (ii) the retailer decides selling price $p_{t}$ after observing the quality level and wholesale price. If the app channel exists, the retailer also decides a selling price $p_{a}$ in the app channel. Then, the profits of the manufacturer and the retailer are realized. Next, we will first study a simply case that the app channel is not opened. Then, the complex case with app channel will be investigated.

\subsubsection{No app channel under manufacturer-led stackelberg model}

Here, we consider the case without app channel. The retailer procures products from the manufacturer and then sells them in the final markets. Backward induction is applied to solve the equilibrium of the game. With the quality level $u$ and wholesale price $w$, the retailer chooses his selling price $p_{t}$ by maximizing his profit

$$
\pi_{t}=\left(p_{t}-w\right)\left(1+u-p_{t}\right) .
$$

Anticipating the retailer's selling price $p_{t}=\frac{1+u+w}{2}$, the manufacturer decides the quality level $u$ and wholesale price $w$ to maximize his profit which is given as

$$
\pi_{m}=w\left(1+u-p_{t}\right)-\frac{1}{2} u^{2} .
$$

The following lemma gives the equilibrium outcomes for the case without app channel. 
Lemma 3.1. At the equilibrium of manufacturer-led stackelberg model without app channel, the opmial wholesale price $w_{f}^{n *}=\frac{2}{3}$, the quality level $u_{f}^{n *}=\frac{1}{3}$, the selling price are $p_{t}^{n *}=1$, and the corresponding profits of the manufacturer and the retailer are given by $\pi_{f m}^{n *}=\frac{1}{6}$ and $\pi_{f t}^{n *}=\frac{1}{9}$.

\subsubsection{Open app channel under manufacturer-led stackelberg model}

In this case, we study the case that the app channel is introduced. The retailer will sell the product through both physical channel and app channel. The retailer's profit is derived from two segments: the first is from the physical channel, and the second is from his app channel. Thus, the profit of the retailer is given as

$$
\begin{aligned}
\pi_{t}= & \left(p_{t}-w\right)\left(1-\frac{p_{t}-\theta p_{a}-(1-\theta) r+(\theta-1) u}{1-\theta}\right) \\
& +\left(p_{a}-w\right)\left(\frac{p_{t}-\theta p_{a}-(1-\theta) r+(\theta-1) u}{1-\theta}-\frac{\theta p_{a}+(1-\theta) r-\theta u}{\theta}\right) .
\end{aligned}
$$

The profit of the manufacturer is given as

$$
\pi_{m}=w\left(1-\frac{\theta p_{a}+(1-\theta) r-\theta u}{\theta}\right)-\frac{1}{2} u^{2} .
$$

According to the above mentioned profit functions of the manufacturer and retailer, we can obtain the equilibrium outcomes of both parties. The results are presented in the following lemma. All proofs are given in the Appendix A.

Lemma 3.2. At the equilibrium of manufacturer-led stackelberg model with app channel, the wholesale price, the quality level, the selling prices for app channel and the physical store are

$$
\begin{aligned}
& w_{f}^{a *}=\frac{(3+\theta)(r(\theta-1)+2 \theta)}{4 \theta(2+\theta)}, \quad u_{f}^{a *}=\frac{r(\theta-1)+2 \theta}{2 \theta(2+\theta)}, \\
& p_{f a}^{*}=\frac{(1+r) \theta-r}{\theta}, \quad p_{f t}^{a *}=\frac{(5+\theta)(r(\theta-1)+2 \theta)}{4 \theta(2+\theta)},
\end{aligned}
$$

respectively, and the corresponding profits of the manufacturer and the retailer are given by

$$
\begin{aligned}
\pi_{f m}^{a *} & =\frac{(r(\theta-1)+2 \theta)^{2}}{8 \theta^{2}(2+\theta)}, \\
\pi_{f t}^{a *} & =\frac{4 r \theta(\theta-1)(3+\theta)+4 \theta^{2}(3+\theta)+r^{2}(1-\theta)(19+\theta(14+3 \theta))}{16 \theta^{2}(2+\theta)^{2}} .
\end{aligned}
$$

\subsection{Retailer-led stackelberg}

In this section, the case that the retailer acts as the leader of supply chain will be studied. The retailer is the stackelberg leader, and the manufacturer responses as the stackelberg follower. Let $s$ denote this case. The timeline of retailer being the leader is as follows: (i) the retailer determines its retail margins $h_{t}$ with $h_{t}=p_{t}-w$. If there exists an app channel, the retailer also decides the margins $h_{t}$ for this channel with $h_{a}=p_{a}-w$; (ii) the manufacturer decides the quality level $u$ and the wholesale price $w$ after observing retail's strategies. Then, the profits of the manufacturer and the retailer are realized. Similar to Section 3.3, we first characterize the case where the app channel is not introduced. Then, we investigate the complex case where the app channel is introduced by the retailer. 


\subsubsection{No app channel under retailer stackelberg-led model}

In this part, we explore the case where the app channel is not introduced with retailer being the Stackelberg leader. Backward induction is applied to obtain the equilibrium outcomes. Given retail margin strategy $h_{t}$, the manufacturer chooses the quality level $u$ and wholesale price $w$ by maximizing his profit

$$
\pi_{m}=w\left(1+u-\left(h_{t}+w\right)\right)-\frac{1}{2} u^{2} .
$$

With anticipating the quality level $u=1-h_{t}$ and wholesale price $w=1-h_{t}$, the retailer decides the retail margin $h_{t}$ to maximize his profit that is given by

$$
\pi_{t}=h_{t}\left(1+u-\left(h_{t}+w\right)\right) .
$$

The equilibrium outcomes of this Stackelberg game is given in the following lemma.

Lemma 3.3. At the equilibrium of retailer-led stackelberg model without app channel, the optimal wholesale price $w_{s}^{n *}=\frac{1}{2}$, the quality level $u_{s}^{n *}=\frac{1}{2}$, the selling price are $p_{t}^{s *}=1$, and the corresponding profits of the manufacturer and the retailer are given by $\pi_{s m}^{n *}=\frac{1}{8}$ and $\pi_{s t}^{n *}=\frac{1}{4}$.

\subsubsection{Open app channel under retailer-led stackelberg model}

In this subsection, we consider that case that the retailer establishes the app channel. Let $h_{a}$ represent the retail margin for the app channel decided by the retailer. We present the profit functions of the manufacturer and the retailer. Similar to (3.5), the manufacturer's profit is given as

$$
\pi_{m}=w\left(1-\frac{(1-\theta) r+\theta\left(h_{a}+w\right)-\theta u}{\theta}\right)-\frac{1}{2} u^{2} .
$$

Similar to (3.4), the profit of the retailer is given as

$$
\begin{aligned}
\pi_{r}= & h_{t}\left(1-\frac{h_{t}+w-\theta\left(h_{a}+w\right)-(1-\theta) r+(\theta-1) u}{1-\theta}\right) \\
& +h_{a}\left(\frac{h_{t}+w-\theta\left(h_{a}+w\right)-(1-\theta) r+(\theta-1) u}{1-\theta}-\frac{(1-\theta) r+\theta\left(h_{a}+w\right)-\theta u}{\theta}\right) .
\end{aligned}
$$

According to the above mentioned profit functions of the manufacturer and retailer, we can get the equilibrium outcomes of both parties. The results are given by the following lemma.

Lemma 3.4. At the equilibrium of retailer-led stackelberg model with app channel, the wholesale price, the quality level, the selling prices for app channel and the physical store are

$$
\begin{aligned}
& w_{s}^{a *}=\frac{r(\theta-1)+2 \theta}{\theta(3+\theta)}, \quad u_{s}^{a *}=\frac{r(\theta-1)+2 \theta}{\theta(3+\theta)}, \\
& p_{s a}^{*}=\frac{\theta(1+r)-r}{\theta}, \quad p_{s t}^{a *}=\frac{2(r(\theta-1)+2 \theta)}{\theta(3+\theta)},
\end{aligned}
$$

respectively, and the corresponding profits of the manufacturer and the retailer are

$$
\begin{aligned}
\pi_{s m}^{a *} & =\frac{(r(\theta-1)+2 \theta)^{2}}{2 \theta^{2}(3+\theta)^{2}} \\
\pi_{s t}^{a *} & =\frac{r^{2}-r(1+r) \theta+(1+r) \theta^{2}}{\theta^{2}(3+\theta)} .
\end{aligned}
$$




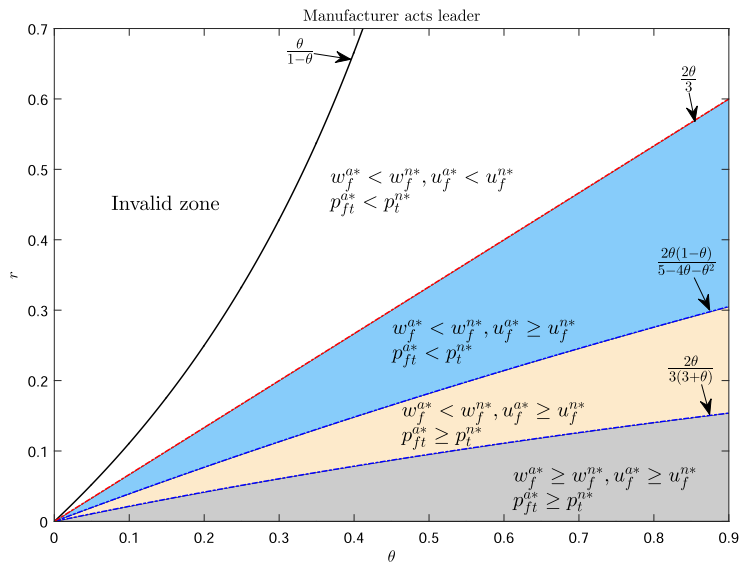

(a)

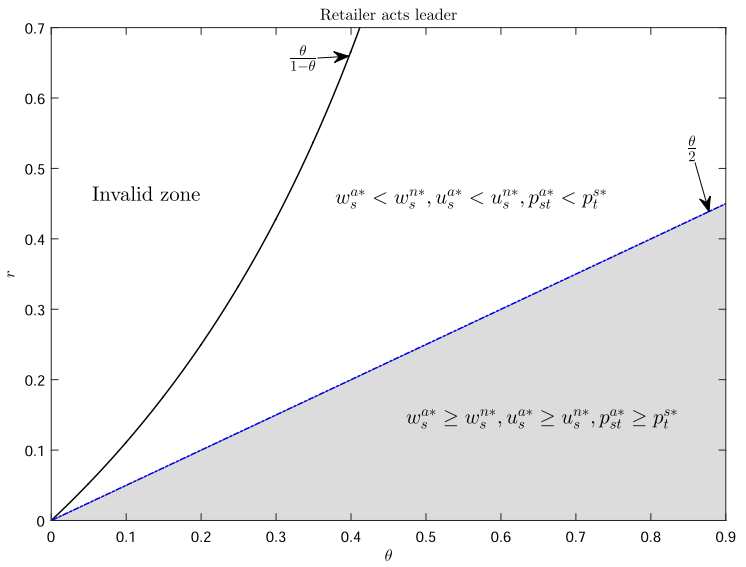

(b)

Figure 1. An illustration of Proposition 4.1.

\section{AnAlysis of the RESUlts}

In this section, we will characterize what are the impacts of adding app channel on the profits of the channel members and total supply chain. The effects of opening app channel on the quality level, wholesale price, selling prices and supply chain member's profits under different power structures will be explored through comparing the equilibrium outcomes of different subgames. Here, we suppose that the retailer firstly determines whether to establish the app channel. This means that whether to open app channel is a longer-term decision. Previous literature has applied the same timing line with modeling choices, such as Jerath and Zhang [14], Abhishek et al. [1] and Xue and Zhang [38].

First, we explore the impacts of adding app channel on the strategies of supply chain members under different power structures. The following proposition describes how the app channel impacts the wholesale price, quality level and selling price for the physical channel.

Proposition 4.1. (i) When the manufacturer acts the leader of the supply chain, for the wholesale price and quality level, we have $w_{f}^{a *} \geq w_{f}^{n *}$ when $0<r \leq \frac{2 \theta}{3(3+\theta)}$, and $w_{f}^{a *}<w_{f}^{n *}$ when $\frac{2 \theta}{3(3+\theta)}<r<\frac{\theta}{1-\theta}$. For the product quality, we have $u_{f}^{a *} \geq u_{f}^{n *}$ when $0<r \leq \frac{2 \theta}{3}$, and $u_{f}^{a *}<u_{f}^{n *}$ when $\frac{2 \theta}{3}<r<\frac{\theta}{1-\theta}$. For the selling price of the physical channel, we have $p_{f t}^{a *} \geq p_{t}^{n *}$ when $0<r \leq \frac{2 \theta(1-\theta)}{5-4 \theta-\theta^{2}}$, and $p_{f t}^{a *}<p_{t}^{n *}$ when $\frac{2 \theta(1-\theta)}{5-4 \theta-\theta^{2}}<r<\frac{\theta}{1-\theta}$.

(ii) When the retailer acts the leader of the supply chain, we have $w_{s}^{a *} \geq w_{s}^{n *}, u_{s}^{a *} \geq u_{s}^{n *}, p_{s t}^{a *} \geq p_{t}^{s *}$ when $0<r \leq \frac{\theta}{2}$, and $w_{s}^{a *}<w_{s}^{n *}, u_{s}^{a *}<u_{s}^{n *}, p_{s t}^{a *}<p_{t}^{s *}$ otherwise.

The results in Proposition 4.1 are depicted in the following figure.

Proposition 4.1 presents insights on how the retailer's app channel affects the wholesale price, product quality and selling price under different power structures. We will start analysis by looking at the results with manufacturer being the leader.

As indicated by Figure 1a, for the manufacturer, more flexibility in adjusting the wholesale price and quality investment is endowed by retailer's ability to add app channel to respond fluctuations of product demand. When the return cost is sufficiently low $\left(r \leq \frac{2 \theta}{3(3+\theta)}\right)$, app channel evokes product quality, and the wholesale price and selling price are adjusted accordingly. The changes are started when the return cost exceeds the threshold $\frac{2 \theta}{3(3+\theta)}$. When $\frac{2 \theta}{3(3+\theta)}<r \leq \frac{2 \theta}{3}$, the wholesale price is decreased although the product quality is promoted. As presented by Arya and Mittendorf [3], in absence of quality decision, adding a new channel with a lower 
product demand will reduce the wholesale price across both channels. When the return cost becomes higher, the effect of decreasing average wholesale price across both channels from adding app channel becomes dominant, thus, the wholesale price is reduced. For the retailer, the selling price is also increased when $\frac{2 \theta}{3(3+\theta)}<r \leq \frac{2 \theta(1-\theta)}{5-4 \theta-\theta^{2}}$. In this region, the return cost is sightly high, thus, facing a decreasing wholesale price and an increasing product quality, the retailer has incentive to set an aggressive selling price to reap profit. When $r$ continuously becomes higher $\left(\frac{2 \theta(1-\theta)}{5-4 \theta-\theta^{2}}<r \leq \frac{2 \theta}{3}\right)$, i.e., the return cost is relatively high, the retailer has to reduce selling price for the physical channel to stimulate demand in order to slash the negative effect of a relatively high return cost. Hence, a lower selling price is applied. When the return cost is sufficiently high, adding app channel induces the manufacturer to reduce product quality, and the wholesale price and selling price are reduced accordingly.

Moreover, Figure 1a gives a new insight that a higher product quality does not necessarily mean a higher wholesale price. Our results indicate that adding app channel can induce the manufacturer to promote the product quality level but charge a lower wholesale price when the return cost is sightly high. This case, however, only exists when the manufacturer acts the leader of the supply chain.

In a retailer-led supply chain, the results are quite different from that in a manufacturer-led supply chain. Figure $1 \mathrm{~b}$ shows that when the cost of returning product is relatively small $\left(r<\frac{\theta}{2}\right)$, the retailer's ability to open app channel can excite a manufacturer's quality investment, and hinder a manufacturer's quality investment otherwise. Accordingly, the wholesale price and selling price are promoted when the cost of returning product is relatively small, and are reduced otherwise. Our results imply that, in a retailer-led supply chain, the trend of prices adjustment is consistent with that of quality adjustment. A higher product quality always means an aggressive wholesale price and selling price.

In addition, it is easily obtained that $\frac{2 \theta}{3}>\frac{\theta}{2}$. This suggests adding app channel is more likely to induce the manufacturer to promote product quality in a manufacturer-led supply chain.

Next, we will identify how the retailer's ability to open app channel affects the profits of the manufacturer and the retailer under different power structures. The results are provided in the following proposition.

Proposition 4.2. (i) When the manufacturer acts the leader, for the manufacturer, we have $\pi_{f m}^{a *} \geq \pi_{f m}^{n *}$ when $0<r \leq \frac{6 \theta-2 \theta \sqrt{3(2+\theta)}}{3(1-\theta)}$, and $\pi_{f m}^{a *}<\pi_{f m}^{n *}$ when $\frac{6 \theta-2 \theta \sqrt{3(2+\theta)}}{3(1-\theta)}<r<\frac{\theta}{1-\theta}$. For the retailer, $\pi_{f t}^{a *}$ is always larger than $\pi_{f t}^{n *}$.

(ii) When the retailer acts the leader, for the manufacturer, we have $\pi_{s m}^{a *} \geq \pi_{s m}^{n *}$ when $0<r \leq \frac{\theta}{2}$, and $\pi_{s m}^{a *}<\pi_{s m}^{n *}$ when $\frac{\theta}{2}<r<\frac{\theta}{1-\theta}$. For the retailer, $\pi_{s t}^{a *}$ is always larger than $\pi_{s t}^{n *}$.

The following figure illustrates how adding app channel affects the manufacturer's profits under different power structures of the supply chain in Proposition 4.2.

Figure 2 illustrates that for the manufacturer and the retailer, adding app channel in a supply chain can result in two distinct profit predictions: win-win, lose-win. In addition, Proposition 4.2 provides the condition under which adding app channel will make both the manufacturer and the retailer better off. We first analysis the retailer's profit. Proposition 4.2 states that the retailer's ability to add app channel always benefits the retailer no matter which power structure of the supply chain is. From the retailer's perspective, although adding app channel evokes channel conflict in the retail market, dual selling channels enable the retailer to implement price discrimination for consumers with different channel preferences. And the retailer always benefits from employing price discrimination.

From Figure 2, however, we find that whether adding app channel benefits the manufacturer or not is conditional on consumers' return cost $r$. According to Lemmas 3.1 and 3.2, we can easily obtain the total product demand $D_{f n}=\frac{1}{3}$ without app channel and $D_{f a}=\frac{2 \theta+r(\theta-1)}{2 \theta(2+\theta)}$ with app channel when the manufacturer acts the leader of the supply chain. Comparing $D_{f n}$ and $D_{f a}$, we can find that $D_{f a} \geq D_{f n}$ when $0<r \leq \frac{2 \theta}{3}$, and $D_{f a}<D_{f n}$ otherwise. Adding app channel can boost product demand only when the return cost is relatively low. In order to clearly explain the results, the following figure comprehensively shows the effects of adding app channel on the product quality, the wholesale price and the profit of the manufacturer. 


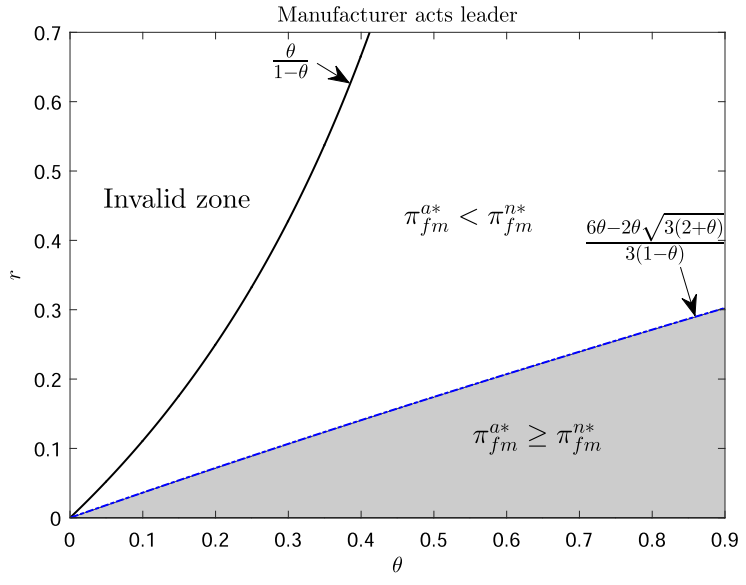

(a)

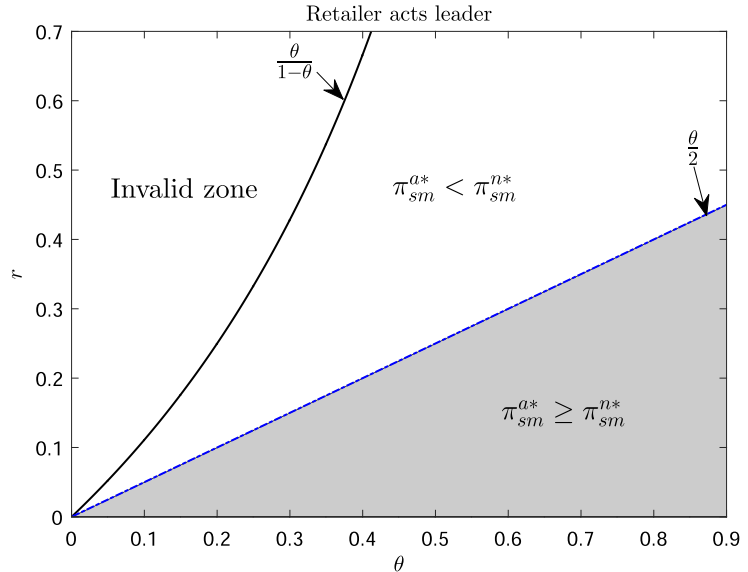

(b)

Figure 2. An illustration of Proposition 4.2.

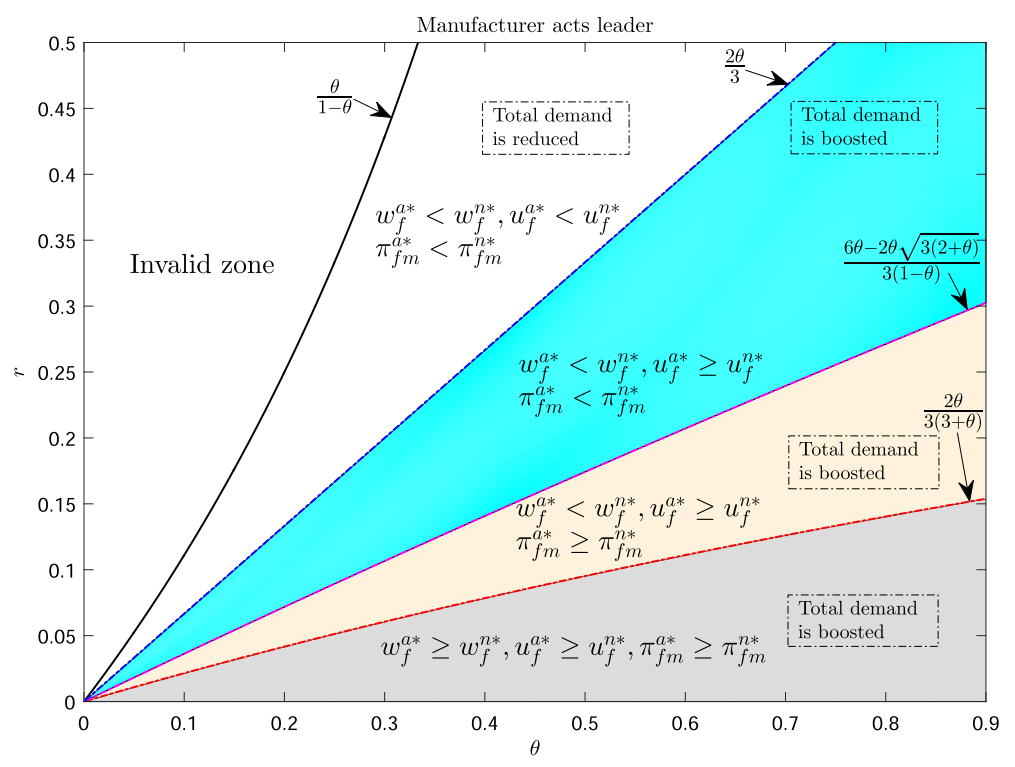

FiguRE 3. An illustration of the effects of adding app channel on the product quality, the wholesale price and the manufacturer's profits.

When $0<r<\frac{2 \theta}{3(3+\theta)}$, i.e., the cost of returning product is relatively low, adding app channel induces the manufacturer to promote product quality and wholesale price simultaneously, naturally, the total product demand is promoted, which benefits the manufacturer. As the cost of returning product is increasing, i.e., $r>\frac{2 \theta}{3(3+\theta)}$, as shown by Figure 3, adding app channel induces the manufacturer to decrease the wholesale price. When $\frac{2 \theta}{3(3+\theta)}<r<\frac{6 \theta-2 \theta \sqrt{3(2+\theta)}}{3(1-\theta)}$, the positive effect of boosting demand dominates the negative effect from reducing wholesale price, thus, the manufacturer is also beneficial from the retailer's ability to add app channel. When the cost of returning product exceeds the threshold $\frac{6 \theta-2 \theta \sqrt{3(2+\theta)}}{3(1-\theta)}$, but is not very high 
$\left(\frac{6 \theta-2 \theta \sqrt{3(2+\theta)}}{3(1-\theta)}<r<\frac{2 \theta}{3}\right)$, the result is reverse. In such circumstances, the negative effect from reducing wholesale price dominates the positive effect of boosting demand from an increasing quality level, thus, adding app channel hurts the manufacturer. It is a counterintuitive result because it proclaims that a boosted demand does not always generate a higher profit. As a result, the manufacturer should be aware of the return cost when facing an app channel established by the retailer. When the return cost is sightly high, more attention should be paid to demand balance because the decreased wholesale price incurred by app channel can weaken the channel profitability. When the cost of returning product is very high $\left(r>\frac{2 \theta}{3}\right)$, adding app channel induces the manufacturer to reduce product quality and wholesale price simultaneously, naturally, the manufacturer's profit is worse off.

When the retailer acts the leader of the supply chain, for the manufacturer, the result is analogous with the case when the manufacturer acts the leader. According to Lemmas 3.3 and 3.4, we can easily obtain the total product demand $D_{s n}=\frac{1}{2}$ without app channel and $D_{s a}=\frac{2 \theta+r(\theta-1)}{\theta(3+\theta)}$ with app channel when the retailer acts the leader. Comparing $D_{s n}$ and $D_{s a}$, we can find that $D_{s a} \geq D_{s n}$ when $0<r \leq \frac{\theta}{2}$, and $D_{s n}>D_{s a}$ otherwise. If $r \leq \frac{\theta}{2}$, as shown by Figure 2b, adding app channel makes the manufacturer better off. Recalling part (ii) in Proposition 4.1, in a retailer-led supply chain, adding app channel induces the manufacturer to promote product quality and wholesale price simultaneously when the cost of returning product is small $\left(r \leq \frac{\theta}{2}\right)$. Thus, the manufacturer is better off resulted from a boosting demand. When the cost of returning product is high $\left(r>\frac{\theta}{2}\right)$, the results are reverse. The manufacturer becomes worse off caused by a reducing demand.

Proposition 2 indicates that, adding app channel can make both manufacturer and retailer better off resulting in a Pareto outcome when consumers' return cost is relatively small. Under this condition, both the manufacturer and the retailer hold an identical attitude towards the app channel. This result differs from that in Zhang et al. [46] which find that the manufacturer can be better off from retailer's directly selling channel with a transfer payment from the retailer in the absence of quality decision. Our results imply that adding app channel is able to make the manufacturer better off without any transfer payment. In addition, it is easily to find that $\frac{\theta}{2}>\frac{6 \theta-2 \theta \sqrt{3(2+\theta)}}{3(1-\theta)}$. This indicates that the region where the manufacturer benefits from adding app channel with retailer being the leader is more vast than the case with manufacturer being the leader. In other words, adding app channel is more likely to result in a win-win situation when the retailer acts the leader of the supply chain.

Proposition 4.2 therefore makes two contributions to the literature. First, we identify a condition under which both the manufacturer and the retailer will gain from app channel without any transfer payment (winwin situation). Second, we show that it is more likely to result in a win-win situation with retailer being the leader.

From Proposition 4.2, we can obtain the following corollary.

Corollary 4.3. The retailer can offer a transfer payment $T$ as specified below to the manufacturer such that such a fee can achieve Pareto improvement for both the manufacturer and the retailer even though adding app channel hurts the manufacturer.

(i) If $r>\frac{6 \theta-2 \theta \sqrt{3(2+\theta)}}{3(1-\theta)}, \pi_{f m}^{n *}-\pi_{f m}^{a *}<T<\pi_{f t}^{a *}-\pi_{f t}^{n *}$ when the manufactuer acts the leader.

(ii) If $r>\frac{\theta}{2}, \pi_{s m}^{n *}-\pi_{s m}^{a *}<T<\pi_{s t}^{a *}-\pi_{s t}^{n *}$ when the retailer acts the leader.

Next, we explore the effect of adding app channel on the profits of whole supply chain under different power structure. Based on the results obtained above, we obtain the following result.

Proposition 4.4. (i) When the manufacturer acts the leader, adding app channel always makes the supply chain better off.

(ii) When the retailer acts the leader, there exists two thresholds $y_{1}=\frac{\theta}{2}$ and $y_{2}=\frac{\theta(13+3 \theta)}{2(7+\theta)}$ such that adding app channel makes the supply chain better off when $0<r \leq y_{1}$ or $y_{2} \leq \theta<\frac{\theta}{1-\theta}$, and adding app channel makes the supply chain worse off when $y_{1}<r<y_{2}$. 


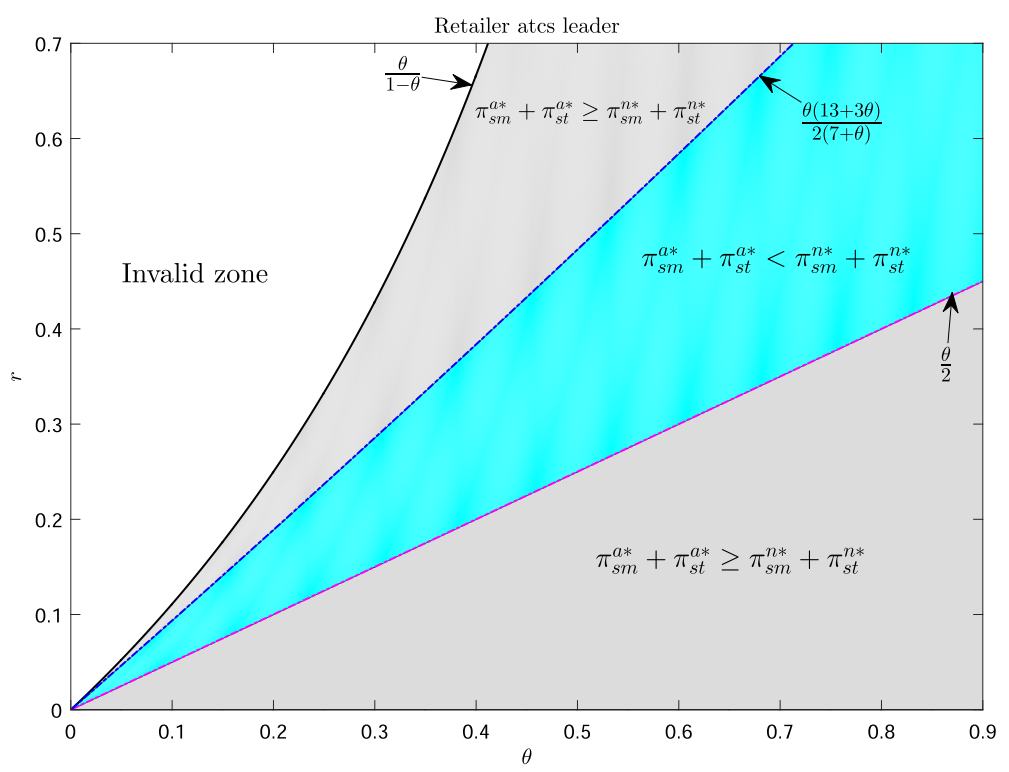

Figure 4. An illustration of part (ii) in Proposition 4.4.

The following figure depicts part (ii) in Proposition 4.4.

Proposition 4.4 shows that the results under different power structures are different. When the manufacturer acts the leader of the supply chain, according to part (i) in Proposition 4.4, the supply chain is always better off with retailer adding app channel. From part (i) in Proposition 4.2, we know that when the return cost is relatively low $\left(r \leq \frac{6 \theta-2 \theta \sqrt{3(2+\theta)}}{3(1-\theta)}\right)$, adding app channel leads to a win-win situation for the manufacturer and the retailer. Clearly, the supply chain is better off from adding app channel. When the the return cost is relatively high $\left(r>\frac{6 \theta-2 \theta \sqrt{3(2+\theta)}}{3(1-\theta)}\right)$, although the manufacturer becomes worse off, the rise of retailer's profit can compensate for the loss of the manufacturer's profit, consequently, the total supply chain also can benefit from opening app channel.

When the retailer acts the leader, from Figure 4, whether the supply chain benefits from app channel or not is conditional on the return cost $r$. When the return cost is relatively low $\left(r \leq \frac{\theta}{2}\right)$ or high $\left(r \geq \frac{\theta(13+\theta)}{2(7+\theta)}\right)$, the supply chain benefits from adding app channel. Recalling Proposition 4.2, when the return cost is relatively low, both the manufacturer and the retailer are better off, naturally, the supply chain is better off. When the return cost is relatively high, the retailer will deepen the degree of price discrimination for consumers with different channel preferences, thus, although the manufacturer suffers from the introduction of app channel, the rise of retailer's profit dominates the loss of the manufacturer's profit, thus, the supply chain still becomes better off. When the return cost is moderate $\left(\frac{\theta}{2}<r<\frac{\theta(13+\theta)}{2(7+\theta)}\right)$, the result is reverse. The loss of the manufacturer's profit outweighs the rise of retailer's profit, then, the supply chain will suffer from adding app channel. As a result, it is advisable to be aware of the return cost in a retailer-led supply chain. When the return cost is moderate $\left(\frac{\theta}{2}<r<\frac{\theta(13+\theta)}{2(7+\theta)}\right)$, the product demand balance needs attention because a decreased demand induced by adding app channel can slash the channel profitability.

The main contribution of Proposition 4.4 is that we identify a condition in which adding app channel will hurt the whole supply chain when the retailer is the leader. Specifically, in a retailer-led supply chain, a relatively low or high return cost will make the whole supply chain better off with adding app channel. Only when the return cost is moderate, the whole supply chain will be suffered from retailer's ability to add app channel. 
Our results suggest that, from the perspective of whole supply chain, the chain members can embrace a high return cost with retailer being the leader.

\section{Extension: Welfare ANALysis}

Next, we will examine how adding app channel affects consumer welfare under different power structures. Denote the consumer welfare as CW. Following Choudhary et al. [10] and Zhang et al. [45], without app channel, we formulate consumer's welfare $\mathrm{CW}$ as

$$
\mathrm{CW}=\int_{p_{t}^{*}-u^{*}}^{1}\left(v+u^{*}-p_{t}^{*}\right) \mathrm{d} v .
$$

According to (5.1), we present a lemma as follows to give consumer welfare without app channel under different power structures.

Lemma 5.1. Without app channel, consumer welfare $\mathrm{CW}_{m}=\frac{1}{18}$ when the manufacturer acts the leader of the supply chain, and $\mathrm{CW}_{t}=\frac{1}{8}$ when the retailer acts the leader.

When the retailer adds the app channel, we formulate consumer welfare $\mathrm{CW}_{a}$ as

$$
\mathrm{CW}_{a}=\int_{\tilde{v}}^{1}\left(v+u^{*}-p_{t}^{*}\right) \mathrm{d} v+\int_{\frac{(1-\theta) r+\theta p_{a}^{*}-\theta u^{*}}{\theta}}^{\tilde{v}}\left(\theta\left(v+u^{*}-p_{a}^{*}\right)-(1-\theta) r\right) \mathrm{d} v .
$$

where $\tilde{v}=\frac{p_{t}^{*}-\theta p_{a}^{*}-(1-\theta) r+(\theta-1) u^{*}}{1-\theta}$.

According to (5.2), the following lemma gives consumer welfare with app channel under different power structures.

Lemma 5.2. With app channel, when the manufacturer acts the leader of the supply chain, consumer welfare is as follows

$$
\mathrm{CW}_{a m}=\frac{4 r(\theta-3)(\theta-1) \theta+4 \theta^{2}(1+3 \theta)-r^{2}(\theta-1)(9+\theta(22+5 \theta))}{32 \theta^{2}(2+\theta)^{2}} .
$$

When the retailer acts the leader of the supply chain, consumer welfare is given as follows

$$
\mathrm{CW}_{a t}=\frac{2 r(\theta-1)^{2} \theta+\theta^{2}(1+3 \theta)+r^{2}(1+(2-3 \theta) \theta)}{2 \theta^{2}(3+\theta)^{2}} .
$$

By comparing consumer welfare presented by Lemmas 5.1 and 5.2 , we can obtain the following results.

Proposition 5.3. (i) When the manufacturer acts the leader of the supply chain, we have $\mathrm{CW}_{a m} \leq \mathrm{CW}_{m}$ if $0<r \leq \frac{6 \theta(\theta-3)+4 \theta(2+\theta) \sqrt{9-5 \theta}}{3(9+\theta(22+5 \theta))}$, and $\mathrm{CW}_{a m}>\mathrm{CW}_{m}$ if $\frac{6(\theta-3) \theta+4 \theta(2+\theta) \sqrt{9-5 \theta}}{3(9+\theta(22+5 \theta))}<r<\frac{\theta}{1-\theta}$.

(ii) When the retailer acts the leader of the supply chain, we have $\mathrm{CW}_{a t} \leq \mathrm{CW}_{t}$ if $0<r \leq \frac{\theta}{2}$, and $\mathrm{CW}_{\text {at }}>\mathrm{CW}_{t}$ if $\frac{\theta}{2}<r<\frac{\theta}{1-\theta}$.

The results in Proposition 5.3 are illustrated by the following figures.

Figure 5a demonstrates that when the return cost is sufficiently low, retailer's ability to add app channel hurts consumers with manufacturer being the leader. Under this condition, an aggressive selling prices are employed by the retailer to grab more profit from consumers, thus, consumer welfare will be worse off. When the return cost becomes high $\left(r>\frac{6 \theta(\theta-3)+4 \theta(2+\theta) \sqrt{9-5 \theta}}{3(9+\theta(22+5 \theta))}\right)$, consumer can be better off from adding app channel. As shown in Figure 5b, the results are analogous in a retailer-led supply chain. When the return cost is relatively low $\left(r<\frac{\theta}{2}\right)$, consumers suffer from the aggressive selling prices, otherwise, opening app channel will make consumer welfare better off. 


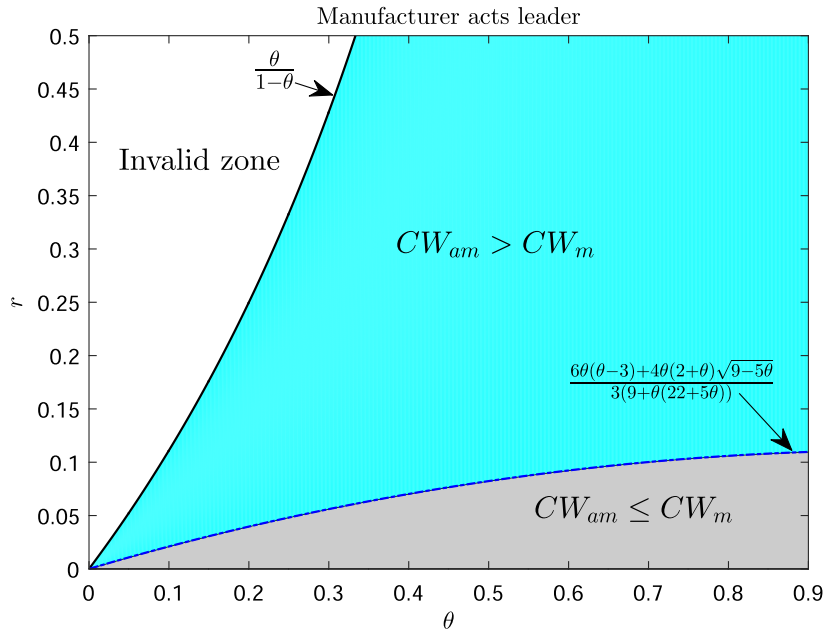

(a)

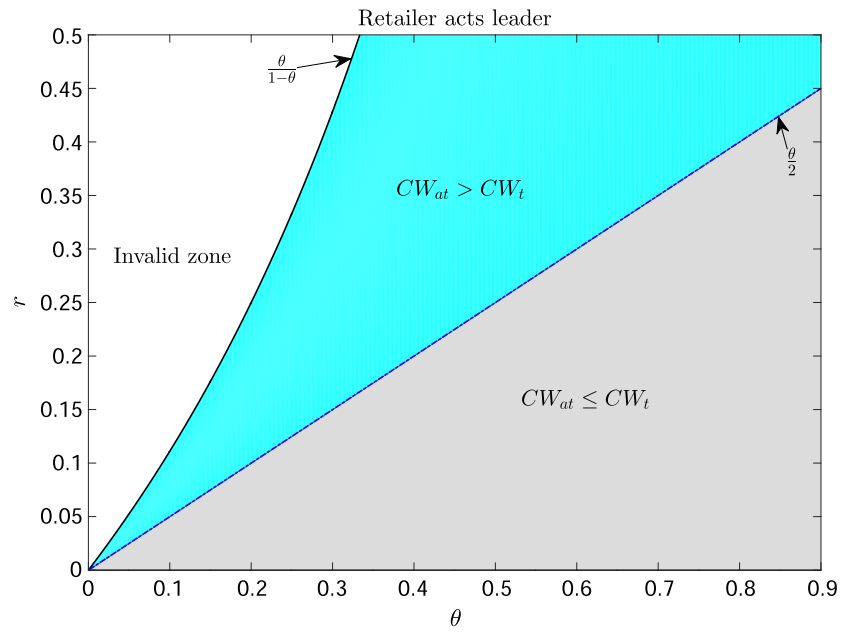

(b)

Figure 5. An illustration of Proposition 5.3.

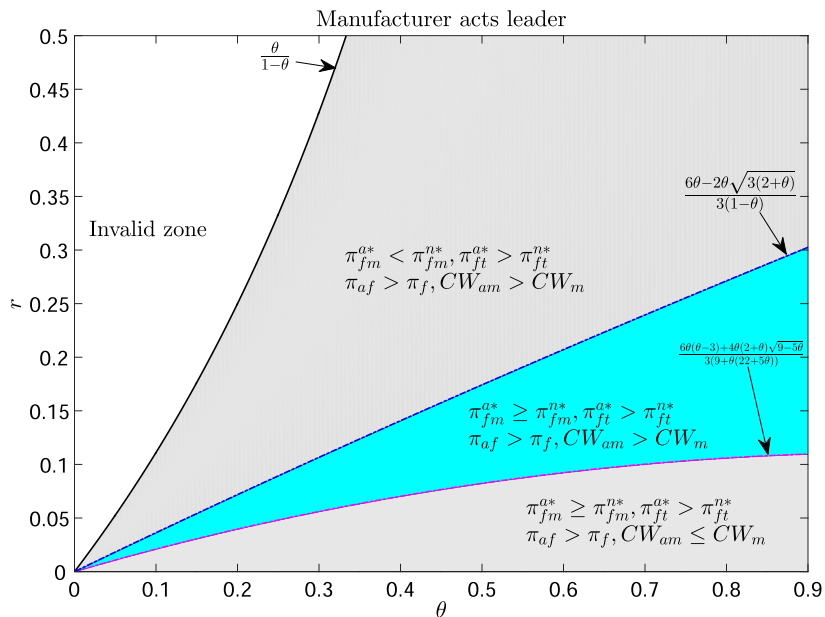

(a)

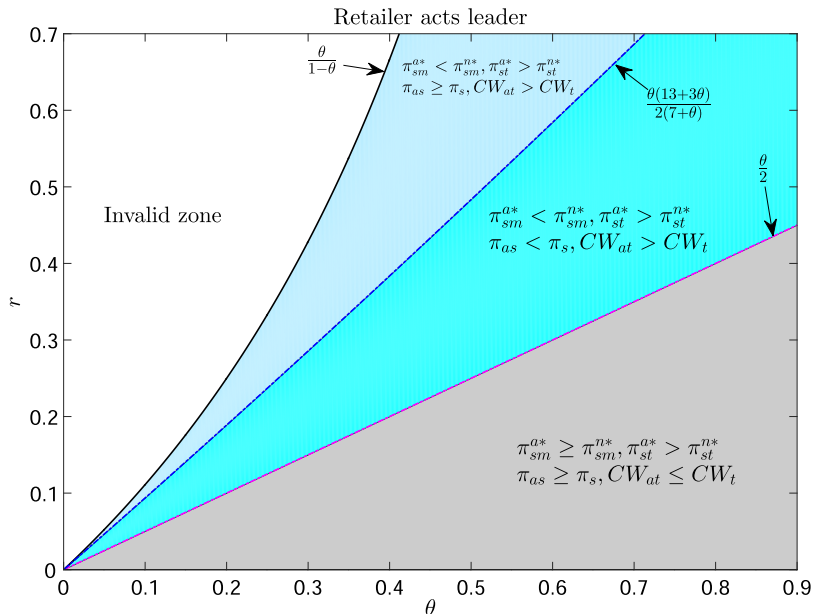

(b)

FIGURE 6. Effects of app channel on profits of the manufacturer, the retailer, the whole supply chain and consumer welfare.

In addition, one can easily obtain $\frac{\theta}{2}>\frac{6 \theta(\theta-3)+4 \theta(2+\theta) \sqrt{9-5 \theta}}{3(9+\theta(22+5 \theta))}$. This implies that consumer welfare is more likely to be better off from adding app channel in a manufacturer-led supply chain.

Combining the results obtained above, is it possible for the manufacturer, the retailer, the whole supply chain and consumer to gain from adding app channel simultaneously under different power structures? If so, under what condition? To answer these questions, we need to comprehensively analyse the results obtained above. We denote $\pi_{f}=\pi_{f m}^{n *}+\pi_{f t}^{n *}, \pi_{a f}=\pi_{f m}^{a *}+\pi_{f t}^{a *}$ when the manufacturer acts leader, and $\pi_{s}=\pi_{s m}^{n *}+\pi_{s t}^{n *}$, $\pi_{a s}=\pi_{s m}^{a *}+\pi_{s t}^{a *}$ when the retailer acts leader. In order to comprehensively analyse the effects of adding app channel on the profits of the manufacturer, the retailer, the whole supply chain and consumer, we summarize the above results in the following figures.

According to Figure 6, we can state the following results. 
TABLE 2. The optimal strategies and profits for the chain members under different power structures.

\begin{tabular}{llllllll}
\hline \multirow{2}{*}{ Parameter } & & \multicolumn{6}{c}{$\theta=0.8$} \\
\cline { 3 - 8 } & & \multicolumn{6}{c}{ The manufacturer-led game } \\
\cline { 3 - 8 } & $w_{f}^{a *}$ & $u_{f}^{a *}$ & $p_{f a}^{*}$ & $p_{f t}^{*}$ & $\pi_{f m}^{a *}$ & $\pi_{f t}^{a *}$ \\
\hline \multirow{4}{*}{$r$} & 0.05 & 0.6743 & 0.3549 & 0.9875 & 1.0354 & 0.1763 & 0.1199 \\
& 0.1 & 0.6701 & 0.3527 & 0.975 & 1.0344 & 0.1741 & 0.1189 \\
& 0.15 & 0.6658 & 0.3504 & 0.9625 & 1.0328 & 0.1719 & 0.1184 \\
& 0.2 & 0.6616 & 0.3482 & 0.95 & 1.0305 & 0.1698 & 0.1183 \\
\hline \multirow{4}{*}{} & & \multicolumn{5}{c}{ The retailer-led game } \\
\cline { 3 - 8 } & & $w_{s}^{a *}$ & $u_{s}^{a *}$ & $p_{s a}^{*}$ & $p_{s t}^{*}$ & $\pi_{s m}^{a *}$ & $\pi_{s t}^{a *}$ \\
\hline & 0.05 & 0.523 & 0.523 & 0.9875 & 1.0461 & 0.1368 & 0.2601 \\
& 0.1 & 0.5197 & 0.5197 & 0.975 & 1.0395 & 0.1351 & 0.2574 \\
& 0.15 & 0.5164 & 0.5164 & 0.9625 & 1.0329 & 0.1334 & 0.2551 \\
& 0.2 & 0.5132 & 0.5132 & 0.95 & 1.0263 & 0.1317 & 0.2533 \\
\hline
\end{tabular}

Claim 5.4. (i) When the manufacturer acts the leader, adding app channel is able to make all the part (the manufacturer, the retailer, the whole supply chain and consumer) better off when $\frac{6 \theta(\theta-3)+4 \theta(2+\theta) \sqrt{9-5 \theta}}{3(9+\theta(22+5 \theta))}<r<\frac{6 \theta-2 \theta \sqrt{3(2+\theta)}}{3(1-\theta)}$.

(ii) When the retailer acts the leader, it is impossible to make all the parts (the manufacturer, the retailer, the whole supply chain and consumers) better off.

We make a few observations on Claim 5.4. Our results reveal that, for the return cost, there exists an interval, namely $\left[\frac{6 \theta(\theta-3)+4 \theta(2+\theta) \sqrt{9-5 \theta}}{3(9+\theta(22+5 \theta))}, \frac{6 \theta-2 \theta \sqrt{3(2+\theta)}}{3(1-\theta)}\right]$, such that the profits of the manufacturer, the retailer, the whole supply chain and consumer welfare are all better off in manufacturer-led supply chain. In other words, when the return cost is neither too small nor too large, it is possible all the parts (the manufacturer, the retailer, the supply chain and consumers) to reach a Pareto improvement from adding app channel when the manufacturer acts the leader. In a retailer-led supply chain, however, all the parts cannot become better off from adding app channel. Our results recommend that the value of return cost should be carefully considered, then, it will have opportunity to improve both profitability and consumers welfare through adding app channel.

Claim 5.4 therefore makes contribution to the literature. It is a new result to identify a condition under which the manufacturer, the retailer, the supply chain and consumers will gain from retailer's ability to add app channel, which may result in a Pareto improvement. This condition only exists in a manufacturer-led supply chain.

\section{NumericAl EXAMPLE}

We give a numerical example to illustrate the presented results with retailer adding the app channel. The sensitivity analysis of parameter $r$ is also given to show how the return cost affects the channel members' strategies under different power structures. The probability that consumers like the product purchased from app channel $\theta$ is taken values of $\theta=0.8$. The parameter value is selected according to the previous studies, such as Jing [17] and Mehra et al. [21]. The results are presented in the following table.

From Table 2, the following results can be observed. (i) The optimal product quality level with the retailer being the supply chain leader is higher than that with the manufacturer being the supply chain leader. This means that the consumers could enjoy a higher quality product when the retailer controls the supply chain. (ii) Both the manufacturer's and the retailer's profits are decreasing with respect to the return cost regardless of 
the power structure. This indicates that, when the app channel is introduced by the retailer, the return cost is harmful to the chain members no matter which power structure of the supply chain is. This result is intuitive.

\section{Conclusion}

It is prevailing for a retailer to add an app channel along with the physical retail channel to sell product. However, the effect of opening app channel on performance of supply chain with quality decision and different power structures' setting are intriguing but unexplored. This gap is filled by our study. With setting the product quality as an endogenous decision, we examine the impacts of adding an app channel on the equilibrium outcomes in a supply chain under different power structures. The novel insights obtained make contribute to the theory of power structure, and can be employed to determine the implications of app channel and the power structure in a supply chain.

The main insights are summarized in the following: First, our findings imply that the effect of adding app channel on the equilibrium outcomes under different power structures of the supply chain is different. Second, we find that, for the manufacturer and retailer, retailer adding app channel in a supply chain can result in two profit situations regardless of the power structure: win-win and lose-win. Adding app channel is able to make the manufacturer better off without any transfer payment. In addition, in a retailer-led supply chain, it is more likely to result in a win-win situation with adding app channel. Third, we present a new result that when the return cost is neither too small nor too large, it is possible for all parts (the manufacturer, the retailer, the supply chain and consumers) to reach a Pareto improvement from adding app channel when the manufacturer acts the leader. This implies that a moderate return cost is sometimes beneficial to profitability and consumer. In summary, these results allow us to understand the role of retailer's app channel in a supply chain with quality decision and different power structures.

There are several research extensions in the future study. First, the retailer may introduce its private label in retailing, which is a part of the final market. It would be of interest to incorporate the private label into model to examine the interaction between online channel and private label. Second, consumers may visit a physical store to experience products first but purchase the products from the online channel, which changes the physical store to be the products' showrooms. This phenomenon is known as showrooming. Future research could take the showrooming effect into consideration to investigate how the showrooming affects the retailer's channel strategy.

\section{Appendix A. Proof of Lemma 3.2}

The backward induction is employed to solve the optimal strategies of the manufacturer and the retailer. First, we calculate the optimal pricing policy for the retailer reacting to the wholesale prince and quality level. We apply the first-order condition to maximize the profit (3.4) w.r.t. the decision variables $p_{t}$ and $p_{a}$, obtaining $p_{f t}=\frac{r(-1+\theta)+\theta(2+2 u+w+w \theta)}{\theta(3+\theta)}$ and $p_{f a}=\frac{\theta(1+u+2 w+\theta+u \theta)+r\left(-2+\theta+\theta^{2}\right)}{\theta(3+\theta)}$. Substituting them into the manufacturer's objective function (3.5), we get

$$
\pi_{f m}=w\left(1-\frac{r(1-\theta)-\theta u+\frac{\theta(1+u+2 w+\theta+u \theta)+r\left(-2+\theta+\theta^{2}\right)}{3+\theta}}{\theta}\right)-\frac{u^{2}}{2} .
$$

The manufacturer chooses $w$ and $q$ to maximize its profit. Applying the first-order condition, we obtain $w_{f}^{a *}=\frac{(3+\theta)(r(\theta-1)+2 \theta)}{4 \theta(2+\theta)}$ and $u_{f}^{a *}=\frac{r(\theta-1)+2 \theta}{2 \theta(2+\theta)}$. Substituting $w_{f}^{a *}$ and $u_{f}^{a *}$ into $p_{f r}$ and $p_{f a}$ yields $p_{f a}^{*}=\frac{(5+\theta)(r(\theta-1)+2 \theta)}{4 \theta(2+\theta)}$ and $p_{f t}^{a *}=\frac{(1+r) \theta-r}{\theta}$. With the optimal wholesale price $w_{f}^{a *}$, the quality level of product $u_{f}^{a *}$ and optimal selling prices $p_{f t}^{*}$ and $p_{f a}^{*}$, we can easily get the optimal profit of the manufacturer $\pi_{f m}^{a *}$ and the optimal profit of the retailer $\pi_{f t}^{a *}$. 


\section{Appendix B. Proof of Lemma 3.4}

In the retailer-led Stackelberg game with adding app channel, the profits of the manufacturer and the retailer are given as (3.6) and (3.7) respectively. The manufacturer first sets $w$ and $u$ to maximize its profit. By solving the first-order conditions, we can obtain

$$
w_{s}^{a}=\frac{\theta\left(1+r-h_{a}\right)-r}{\theta}, \quad u_{s}^{a}=\frac{\theta\left(1+r-h_{a}\right)-r}{\theta},
$$

where $h_{a}$ is the retail margin of the app channel set by the retailer.

Substituting equations (B.1) into (3.7), we can obtain the retailer's profit given as

$$
\pi_{s t}^{a}=h_{t}\left(1+r-h_{a}\right)+\frac{\left(h_{a}-h_{t}\right)^{2}}{\theta-1}-\frac{r h_{a}}{\theta},
$$

where $h_{t}$ is the retail margin of the physical channel set by the retailer.

The retailer set the retail margin $h_{t}$ and $h_{a}$ to maximize her profits. Employing the first-order condition yields

$$
h_{t}^{*}=\frac{r(-1+\theta)+2 \theta}{\theta(3+\theta)}, \quad h_{a}^{*}=\frac{\theta+\theta^{2}+r\left(-2+\theta+\theta^{2}\right)}{\theta(3+\theta)} .
$$

Substituting equations (B.3) into (B.1), we can get $w_{s}^{a *}$ and $u_{s}^{a *}$. Then, we can obtain that the optimal selling prices for the physical channel and app channel are $p_{s t}^{a *}=\frac{2(r(\theta-1)+2 \theta)}{\theta(3+\theta}$ and $p_{s a}^{*}=\frac{\theta(1+r)-r}{\theta}$. With the optimal wholesale price $w_{s}^{a *}$, the quality level $u_{s}^{a *}$ and optimal selling prices $p_{s t}^{a *}$ and $p_{s a}^{*}$, we can easily get the optimal profit of the manufacturer $\pi_{s m}^{a *}$ and the optimal profit of the retailer $\pi_{s t}^{a *}$.

\section{Appendix C. Proof of Proposition 4.1}

We first compare the strategies with app channel to that without app channel under manufacturer-led setting. For the wholesale prices, it can be calculated that $w_{f}^{a *}-w_{f}^{n *}=\frac{1-\theta)(3 r(3+\theta)-2 \theta)}{12 \theta(2+\theta)}$. Since $0<\theta<1$ and $0<r<\frac{\theta}{1-\theta}$, thus, it can be obtained a threshold $\frac{2 \theta}{3(3+\theta)}$ such that $w_{f}^{a *} \geq w_{f}^{n *}$ when $0<r \leq \frac{2 \theta}{3(3+\theta)}$, and $w_{f}^{a *}<w_{f}^{n *}$ when $\frac{2 \theta}{3(3+\theta)}<r<\frac{\theta}{1-\theta}$.

For the product quality, it can be calculated that $u_{f}^{a *}-u_{f}^{n *}=\frac{2 \theta-2 \theta^{2}+r(-3+3 \theta)}{(6 \theta(+\theta)}$. One can easily obtain that $u_{f}^{a *} \geq u_{f}^{n *}$ when $0<r \leq \frac{2 \theta}{3}$, and $u_{f}^{a *}<u_{f}^{n *}$ when $\frac{2 \theta}{3}<r<\frac{\theta}{1-\theta}$.

For the selling prices, we have $p_{f t}^{a *}-p_{t}^{n *}=\frac{2 \theta-2 \theta^{2}+r\left(-5+4 \theta+\theta^{2}\right)}{4 \theta(2+\theta}$. We can obtain $p_{f t}^{a *} \geq p_{t}^{n *}$ when $0<r \leq$ $\frac{2 \theta(1-\theta)}{5-4 \theta-\theta^{2}}$, and $p_{f t}^{a *}<p_{t}^{n *}$ when $\frac{2 \theta(1-\theta)}{5-4 \theta-\theta^{2}}<r<\frac{\theta}{1-\theta}$.

Since $0<r<\frac{\theta}{1-\theta}$, it is easily obtain that $\frac{2 \theta}{3}>\frac{2 \theta(1-\theta)}{5-4 \theta-\theta^{2}}>\frac{2 \theta}{3(3+\theta)}$ by comparing them.

Next, we compare the strategies with app channel to that without app channel under retailer-led setting. One can easily obtain that $w_{s}^{a *}-w_{s}^{n *}=\frac{(1-\theta)(\theta-2 r)}{2 \theta(3+\theta)}, u_{s}^{a *}-u_{s}^{n *}=\frac{\theta-\theta^{2}+r(-2+2 \theta)}{2 \theta(3+\theta)}, p_{s t}^{a *}-p_{t}^{s *}=\frac{(\theta-2 r)(1-\theta)}{2 \theta(3+\theta)}$. Thus, we can obtain that $w_{s}^{a *} \geq w_{s}^{n *}, u_{s}^{a *} \geq u_{s}^{n *}, p_{s t}^{a *} \geq p_{t}^{s *}$ when $0<r \leq \frac{\theta}{2}$, and $w_{s}^{a *}<w_{s}^{n *}, u_{s}^{a *}<u_{s}^{n *}, p_{s t}^{a *}<p_{t}^{s *}$ when $\frac{\theta}{2}<r<\frac{\theta}{1-\theta}$.

\section{Appendix D. Proof of Proposition 4.2}

When the manufacturer acts the leader of the supply chain, it can be calculated that $\pi_{f m}^{a *}-\pi_{f m}^{n *}=$ $\frac{(1-\theta)\left(3 r^{2}-3 r(4+r) \theta+4 \theta^{2}\right)}{24 \theta^{2}(2+\theta)}$. It can be seen that $\pi_{f m}^{a *}-\pi_{f m}^{n *}$ is signed by the part $3 r^{2}-3 r(4+r) \theta+4 \theta^{2}$. Denote $f_{1}(r)=r^{2}-3 r(4+r) \theta+4 \theta^{2}$. Note that $f_{1}(r)$ is convex in $r$, and its symmetry axis is $\frac{2 \theta}{1-\theta}$. Solving the root 
gives a threshold $r_{1}=\frac{6 \theta-2 \theta \sqrt{3(2+\theta)}}{3(1-\theta)}$ such that $\pi_{f m}^{a *} \geq \pi_{f m}^{n *}$ when $0<r \leq \frac{6 \theta-2 \theta \sqrt{3(2+\theta)}}{3(1-\theta)}$, and $\pi_{f m}^{a *}<\pi_{f m}^{n *}$ when $\frac{6 \theta-2 \theta \sqrt{3(2+\theta)}}{3(1-\theta)}<r<\frac{\theta}{1-\theta}$.

For the retailer' profits, we have

$$
\pi_{f t}^{a *}-\pi_{f t}^{n *}=\frac{(1-\theta)\left(-36 r \theta(3+\theta)+4 \theta^{2}(11+4 \theta)+9 r^{2}(19+\theta(14+3 \theta))\right)}{144 \theta^{2}(2+\theta)^{2}} .
$$

It is noted that $\pi_{f t}^{a *}-\pi_{f t}^{n *}$ is signed by the part $-36 r \theta(3+\theta)+4 \theta^{2}(11+4 \theta)+9 r^{2}(19+\theta(14+3 \theta))$. Denote $f_{2}(r)=-36 r \theta(3+\theta)+4 \theta^{2}(11+4 \theta)+9 r^{2}(19+\theta(14+3 \theta))$ which is convex in $r$. Since $0<r<\frac{\theta}{1-\theta}$, one can obtain that $f_{2}(r)>0$. Thus, we have $\pi_{f t}^{a *}$ is always larger than $\pi_{f t}^{n *}$.

When the retailer acts the leader, we have $\pi_{s m}^{a *}-\pi_{s m}^{n *}=\frac{(1-\theta)\left(r^{2}(4-4 \theta)-6 r \theta+7 \theta^{2}+\theta^{3}\right.}{8 \theta^{2}(3+\theta)^{2}}$. Denote $f_{3}(r)=r^{2}(4-4 \theta)-$ $6 r \theta+7 \theta^{2}+\theta^{3}$ which is convex in $r$. Note that the sign of $\pi_{s m}^{a *}-\pi_{s m}^{n *}$ is dependent on $f_{3}(r)$. Solving the root gives a threshold $r_{2}=\frac{\theta}{2}$ such that $\pi_{s m}^{a *} \geq \pi_{s m}^{n *}$ when $0<r \leq \frac{\theta}{2}$, and $\pi_{s m}^{a *}<\pi_{s m}^{n *}$ when $\frac{\theta}{2}<r<\frac{\theta}{1-\theta}$.

For the retailer' profits, we have $\pi_{s t}^{a *}-\pi_{s t}^{n *}=\frac{(1-\theta)(-2 r+\theta)^{2}}{4 \theta^{2}(3+\theta)}>0$. Thus, $\pi_{s t}^{a *}$ is always larger than $\pi_{s t}^{n *}$.

\section{Appendix E. Proof of Corollary 4.3}

In a manufacturer-led supply chain, if the retailer pays a transfer payment $T$ to the manufacturer in order to induce the manufacturer to accept the adding app channel when the return cost $r>\frac{6 \theta-2 \theta \sqrt{(3(2+\theta))}}{3(1-\theta)}$, the following conditions should be satisfied

$$
\left\{\begin{array}{l}
\pi_{f m}^{a *}+T>\pi_{f m}^{n *} \\
\pi_{f t}^{a *}-T>\pi_{f t}^{n *}
\end{array}\right.
$$

Solving this inequations, we obtain $\pi_{f m}^{n *}-\pi_{f m}^{a *}<T<\pi_{f t}^{a *}-\pi_{f t}^{n *}$ so that such a fee can achieve Pareto improvement for both the manufacturer and the retailer even though adding app channel hurts the manufacturer.

In a retailer-led supply chain, the proof is similar to the manufacturer-led case, thus, we omit is here.

\section{Appendix F. Proof of Proposition 4.4}

First, we show that the whole supply chain is always better off in a manufacturer-led supply chain. It can be calculated that

$$
\pi_{f m}^{a *}+\pi_{f t}^{a *}-\left(\pi_{f m}^{n *}+\pi_{f t}^{n *}\right)=\frac{(1-\theta)\left(-36 r \theta(7+3 \theta)+4 \theta^{2}(23+10 \theta)+9 r^{2}(23+\theta(12+\theta))\right)}{144 \theta^{2}(2+\theta)^{2}} .
$$

Since $0<r<\frac{\theta}{1-\theta}$, we can easily obtain that $-36 r \theta(7+3 \theta)+4 \theta^{2}(23+10 \theta)+9 r^{2}(23+\theta(12+\theta))>0$. Thus, $\pi_{f m}^{a *}+\pi_{f t}^{a *}>\pi_{f m}^{n *}+\pi_{f t}^{n *}$, implying adding app channel always makes the supply chain better off when the manufacturer acts the leader.

Next, we consider the case in retailer-led supply chain. It can be calculated that

$$
\pi_{s m}^{a *}+\pi_{s t}^{a *}-\left(\pi_{s m}^{n *}+\pi_{s t}^{n *}\right)=\frac{(1-\theta)\left(4 r^{2}(7+\theta)+\theta^{2}(13+3 \theta)+r(-2 \theta(7+\theta)-2 \theta(13+3 \theta))\right)}{8 \theta^{2}(3+\theta)^{2}} .
$$

Denote $f_{4}(r)=4 r^{2}(7+\theta)+\theta^{2}(13+3 \theta)+r(-2 \theta(7+\theta)-2 \theta(13+3 \theta))$ that is convex in $r$. Solving the root gives two thresholds $y_{1}=\frac{\theta}{2}$ and $y_{2}=\frac{\theta(13+3 \theta)}{2(7+\theta)}$ such that $\pi_{s m}^{a *}+\pi_{s t}^{a *} \geq \pi_{s m}^{n *}+\pi_{s t}^{n *}$ when $0<r \leq y_{1}$ or $y_{2} \leq \theta<\frac{\theta}{1-\theta}$, and $\pi_{s m}^{a *}+\pi_{s t}^{a *}<\pi_{s m}^{n *}+\pi_{s t}^{n *}$ when $y_{1}<r<y_{2}$. This implies that adding app channel makes the supply chain better off when $0<r \leq y_{1}$ or $y_{2} \leq \theta<\frac{\theta}{1-\theta}$, and adding app channel makes the supply chain worse off otherwise. 


\section{Appendix G. Proof of Proposition 5.3}

According to Lemmas 5.1 and 5.2, in a manufacturer-led supply chain, we have

$$
\mathrm{CW}_{a m}-\mathrm{CW}_{m}=\frac{(1-\theta)\left(-36 r(-3+\theta) \theta+4 \theta^{2}(-7+4 \theta)+9 r^{2}(9+\theta(22+5 \theta))\right)}{288 \theta^{2}(2+\theta)^{2}} .
$$

Denote $f_{5}(r)=-36 r(-3+\theta) \theta+4 \theta^{2}(-7+4 \theta)+9 r^{2}(9+\theta(22+5 \theta))$ that is convex in $r$. Solving the root gives a threshold $k_{1}=\frac{6 \theta(\theta-3)+4 \theta(2+\theta) \sqrt{9-5 \theta}}{3(9+\theta(22+5 \theta))}$ such that $\mathrm{CW}_{a m} \leq \mathrm{CW}_{m}$ if $0<r \leq k_{1}$, and $\mathrm{CW}_{a m}>\mathrm{CW}_{m}$ if $k_{1}<r<\frac{\theta}{1-\theta}$.

In a retailer-led supply chain, we have

$$
\mathrm{CW}_{a t}-\mathrm{CW}_{t}=\frac{(1-\theta)\left(2 r^{2}(2+6 \theta)+r(2(5-\theta) \theta-\theta(2+6 \theta))-(5-\theta) \theta^{2}\right)}{8 \theta^{2}(3+\theta)^{2}} .
$$

Denote $f_{6}(r)=r^{2}(2+6 \theta)+r(2(5-\theta) \theta-\theta(2+6 \theta))-(5-\theta) \theta^{2}$ that is convex in $r$. Solving the root gives a threshold $k_{2}=\frac{\theta}{2}$ such that $\mathrm{CW}_{a t} \leq \mathrm{CW}_{t}$ if $0<r \leq \frac{\theta}{2}$, and $\mathrm{CW}_{a t}>\mathrm{CW}_{t}$ if $\frac{\theta}{2}<r<\frac{\theta}{1-\theta}$.

Acknowledgements. This work was supported by the Tianjin Philosophy and Social Sciences Planning Year Project No. TJGLQN17-010.

\section{REFERENCES}

[1] V. Abhishek, K. Jerath, and Z.J. Zhang, Agency selling or reselling? Channel structures in electronic retailing. Manage. Sci. 62 (2015) 2259-2280.

[2] J. Alba and J. Lynch, Interactive home shopping: Consumer, retailer, and manufacturer incentives to participate in electronic marketplaces. J. Mark. 61 (1997) 38-53.

[3] A. Arya and B. Mittendorf, Input price discrimination when buyers operate in multiple markets. J. Ind. Econ. 58 (2010) 846-867.

[4] J. Avery, T.J. Steenburgh, J. Deighton, and M. Caravella, Adding bricks to clicks: predicting the patterns of cross-channel elasticities over time. J. Mark. 76 (2012) 96-111.

[5] A. Balakrishnan, S. Sundaresan, and B. Zhang, Browse-and-Switch: Retail-online competition under value uncertainty. Prod. Oper. Manage. 23 (2014) 1129-1145.

[6] Celine Delacharlerie, Leading the way in online to offline: Leyou's success story. https://www.innovationiseverywhere.com/ leading-the-way-in-online-to-offline-leyous-success-story (2015).

[7] C.L. Chen, Design for the environment: a quality-based model for green product development. Manage. Sci. 47 (2001) $250-263$.

[8] X. Chen, X.J. Wang, and X.k. Jiang, The impact of power structure on the retail service supply chain with an O2O mixed channel. J. Oper. Res. Soc. 67 (2016) 294-301.

[9] J. Chen, L. Liang, D.Q. Yao, and S. Sun, Price and quality decisions in dual-channel supply chains. Eur. J. Oper. Res. 259 (2017) 935-948.

[10] V. Choudhary, A. Ghose, T. Mukhopadhyay, and U. Rajan, Personalized pricing and quality differentiation. Manage. Sci. 51 (2005) 1120-1130.

[11] R. Dai, J.X. Zhang, and W.S. Tang, Cartelization or Cost-sharing? Comparison of cooperation modes in a green supply chain. J. Cleaner Prod. 156 (2017) 159-173.

[12] A. El-Ansary, L. Stern, Power measurement in the distribution channel. J. Mark. Res. 9 (1972) 47-52.

[13] W. Huang and J.M. Swaminathan, Introduction of a second channel: Implications for pricing and profits. Eur. J. Oper. Res. 194 (2009) 258-279.

[14] K. Jerath and Z.J. Zhang, Store within a store. J. Mark. Res. 47 (2010) 748-763.

[15] K. Jerath, S.H. Kim, and R. Swinney, Product quality in a distribution channel with inventory risk. Mark. Sci. 36 (2017) 747-761.

[16] B. Jing, Behavior-based pricing, production efficiency, and quality differentiation. Manage. Sci. 63 (2016) $2049-2395$.

[17] B. Jing, Showrooming and webrooming: information wxternalities between online and offline sellers. Mark. Sci. 37 (2018) 333-506.

[18] D. Kuksov and Y.F. Lin, Information provision in a vertically differentiated competitive marketplace. Mark. Sci. 29 (2010) 122-138.

[19] S.J. Li and A.J. Liu, Pricing strategies in dual-channel supply chain with retailer direct marketing. Adv. Mater. Res. 933 (2014) 902-906. 
[20] Q. Liu and S. Shum, Pricing and capacity rationing with customer disappointment aversion. Prod. Oper. Manage. 22 (2013) $1269-1286$.

[21] A. Mehra, S. Kumar, and J.S. Rajuc, Competitive strategies for brick-and-mortar stores to counter "Showrooming". Manage. Sci. 64 (2018) 3076-3090.

[22] U. Mishra, An inventory model for deteriorating items under trapezoidal type demand and controllable deterioration rate. Prod. Eng. Res. Dev. 9 (2015) 351-365.

[23] U. Mishra, An inventory model for controllable probabilistic deterioration rate under shortages. Evolving Syst. 7 (2016) 287-307.

[24] U. Mishra and C.K. Tripathy, An inventory model for Weibull deteriorating items with salvage value. Int. J. Logist. Syst. Manage., 22 (2015) 67-76.

[25] R.H. Niu, X. Zhao, I. Castillo and T. Joro, Pricing and inventory strategies for a two-stage dual-channel supply chain. Asia-Pac. J. Oper. Res. 29 (2012) 1240004-38.

[26] B.Z. Niu, Z.H. Mu and B.X. Li, O2O results in traffic congestion reduction and sustainability improvement: analysis of "Online-to-Store" channel and uniform pricing strategy. Transp. Res. Part E 122 (2019) 481-505.

[27] H.Y. Shi, Y.C. Liu and N.C. Petruzzi, Consumer heterogeneity, product quality, and distribution channels. Manage. Sci. 59 (2013) 1162-1176.

[28] R.X. Shi, J. Zhang and J. Run, Impact of power structure on supply chains with uncertain demand. Prod. Oper. Manage. 22 (2013) 1232-1249.

[29] J.D. Shulman and X.J. Geng, Add-on pricing by asymmetric firms. Manage. Sci. 59 (2013) 899-917.

[30] J.D. Shulman, A.T. Coughlan and R. Canan Savaskan, Managing consumer returns in a competitive environment. Manage. Sci. 57 (2011) 347-362.

[31] S. Shum, S.L. Tong and T.T. Xiao, On the impact of uncertain cost reduction when selling to strategic customers. Manage. Sci. 63 (2016) 843-860.

[32] Z.Y. Tao, Q.L. Gou and J.Z. Zhang, A local sellers app channel strategy concerning delivery. Int. J. Prod. Res. 58 (2020) $220-255$.

[33] A.A. Tsay and N. Agrawal, Modeling conflict and coordination in multichannel distribution systems: a review. In: Handbook of Quantitative Supply Chain Analysis. Springer (2004) 557-606.

[34] D.W. Wallace, J.L. Giese and J.L. Johnson, Customer retailer loyalty in the context of multiple channel strategies. J. Retailing 80 (2004) 249-263.

[35] X. Xu, Optimal price and product quality decisions in a distribution channel. Manage. Sci. 55 (2009) 1347-1352.

[36] Y. Xu, H. Gurnani and R. Desiraju, Strategic supply chain structure design for a proprietary component manufacturer. Prod. Oper. Manage. 19 (2010) 371-389.

[37] M.S. Xue and J.X. Zhang, Impacts of heterogeneous environment awareness and power structure on green supply chain. RAIRO: OR 52 (2018) 143-157.

[38] M.S. Xue and J.X. Zhang, Supply chain encroachment with quality decision and different power structures. RAIRO: OR 54 (2020) 693-718.

[39] W. Xue, O.C. Demirag and B.Z. Niu, Supply chain performance and consumer surplus under alternative structures of channel dominance. Eur. J. Oper. Res. 239 (2014) 130-145.

[40] M. Yu, L. Debo and R. Kapuscinski, Strategic waiting for consumer-generated quality information: dynamic pricing of new experience goods. Manage. Sci. 62 (2016) 410-435.

[41] J. Zhang, China's online retail sales near $\$ 1 \mathrm{t}$ mark in 2017. http://www.chinadaily.com.cn/business/2017-11/14/ content34521308.htm (2017).

[42] D. Zhang and W.L. Cooper, Managing clearance sales in the presence of strategic customers. Prod. Oper. Manage. 17 (2008) 416-431.

[43] R. Zhang, B. Liu and W.L. Wang, Pricing decisions in a dual channels system with different power structures. Econ. Modell. 29 (2012) 523-533.

[44] L. Zhang, J. Wang and J. You, Consumer environmental awareness and channel coordination with two substitutable products. Eur. J. Oper. Res. 241 (2015) 63-73.

[45] T. Zhang, G. Li, T.C.E. Cheng and K.K. Lai, Welfare economics of review information: Implications for the online selling platform owner. Int. J. Prod. Econ. 184 (2017) 69-79.

[46] P. Zhang, Y. He and C.M. Shi, Retailers channel structure choice: online channel, offline channel, or dual channels? Int. J. Prod. Econ. 191 (2017) 37-50.

[47] Q. Zhang, W.S. Tang, G. Zaccour and J.X. Zhang, Should a manufacturer give up pricing power in a vertical informationsharing channel? Eur. J. Oper. Res. 276 (2019) 910-928.

[48] J.H. Zhou, R.J. Zhao and W.S. Wang, Pricing decision of a manufacturer in a dual-channel supply chain with asymmetric information. Eur. J. Oper. Res. 278 (2019) 809-820.

[49] R.J. Zhu and M.S. Xue, Two-period pricing strategies in a two-echelon supply chain with conspicuous consumption. RAIRO: OR 53 (2018) 667-685. 